\title{
Les sciences au service de l'économie : stratégies gouvernementales en Grande-Bretagne et en France au début du XVIIIe siècle
}

Sciences attending economy: comparative governmental choices in France and Britain in the beginning of the XVIIIth century

Christiane Demeulenaere-Douyère et David J. Sturdy

\section{(2) OpenEdition}

Journals

\section{Édition électronique}

URL : http://journals.openedition.org/dht/1467

DOI : $10.4000 /$ dht. 1467

ISSN : $1775-4194$

Éditeur :

Centre d'histoire des techniques et de l'environnement du Cnam (CDHTE-Cnam), Société des élèves du CDHTE-Cnam

\section{Édition imprimée}

Date de publication : 1 décembre 2010

Pagination : 223-228

ISBN : 978-2-9530779-5-7

ISSN : 0417-8726

\section{Référence électronique}

Christiane Demeulenaere-Douyère et David J. Sturdy, «Les sciences au service de l'économie : stratégies gouvernementales en Grande-Bretagne et en France au début du XVIIIe siècle », Documents pour l'histoire des techniques [En ligne], 19 | 2e semestre 2010, mis en ligne le 21 juin 2011, consulté le 08 septembre 2020. URL : http://journals.openedition.org/dht/1467 ; DOI : https://doi.org/10.4000/ dht. 1467 


\title{
Les sciences au service de l'économie : stratégies gouvernementales en Grande-Bretagne et en France au début du XVIII' siècle
}

\author{
Christiane Demeulenaere-Douyère \\ Archives nationales (site de Paris) \\ chercheur associé CDHTE-Cnam \\ David J. Sturdy $\dagger$ * \\ Université d'Ulster
}

\begin{abstract}
$\bigwedge$ u début du XVIIIle siècle, le postulat que les sciences Asont susceptibles d'apporter des améliorations aux conditions de vie des hommes sur de nombreux plans est incontesté, et l'idée qu'elles peuvent contribuer à faire avancer les intérêts économiques de l'État est également admise. Dans les années 1714-1715, les gouvernements de la Grande-Bretagne et de la France - comme ceux d'autres pays d'ailleurs - se trouvent confrontés avec des problèmes financiers et économiques très considérables à la suite des guerres qui se sont déroulées, presque sans interruption, depuis 1689. Cet article va tenter d'étudier, dans une approche comparative, comment ces deux royaumes, la Grande-Bretagne et la France, ont adopté des stratégies différentes, voire opposées, quant à la mobilisation des sciences et des techniques au service de la rénovation économique.
\end{abstract}

\section{Des conditions politiques difficiles}

Il faut en premier lieu souligner une similitude entre la situation politique de la Grande-Bretagne et celle de la France, à cette époque. Dans chacun de ces deux pays, la tâche de la restauration économique est compliquée par certains événements d'ordre politique.

En Grande-Bretagne, non seulement I'union avec I'Écosse, décidée en 1707, n'est pas encore achevée (il

* Le Pr. David J. Sturdy est décédé brutalement le 5 avril 2009. II était un fin connaisseur de I'histoire de la France des XVII et XVIII siècles; il avait aussi consacré plusieurs travaux à I'Académie royale des sciences de Paris et préparait, au moment de sa disparition, une biographie de l'abbé Bignon, dont il était reste, par exemple, à harmoniser les monnaies et les poids et mesures), mais, en 1714 , le royaume doit faire face à une crise de succession au trône.

Le $1^{\text {er }}$ août 1714, en effet, la reine Anne meurt sans héritier direct et sa succession est disputée entre deux candidats: George, électeur de Hanovre, arrière petit-fils de Jacques $I^{\text {er }}$ et protestant, et James Stuart (connu en France sous le titre de "chevalier de Saint-Georges »), fils de Jacques II et catholique. La cause protestante triomphe, mais l'arrivée de George à Londres provoque une révolte jacobite en Écosse², qui fait peser sur la Grande-Bretagne la menace d'une nouvelle guerre civile (il faut se rappeler que la grande guerre civile des années 1640 fut provoquée par une révolte en Écosse). Au cours des campagnes militaires, les Jacobites, qui sont privés de I'aide militaire de la France, subissent des échecs décisifs

un des spécialistes reconnus. C'était un chercheur de grande érudition et un collègue de grande qualité humaine à la mémoire duquel je tiens à rendre ici un hommage particulier (Christiane Demeulenaere-Douyère).

1 P. W. J. Riley, The Union of England and Scotland: a study in Anglo-Scottish politics of the eighteenth century, Manchester, Manchester University Press, 1978 ; idem, The English ministers and Scotland, 1707-1727, London, Athlone Press, 1964 ; Daniel Szechi, Jacobitism and Tory politics, 1710-1714, Edinburgh, Donald, 1984 ; Christopher A. Whatley, Bought and sold for English gold? Explaining the Union of 1707, East Linton, Tickwell Press, 2001, 2e éd.

2 Sur George ler en Angleterre, Ragnhild Hatton, George I: Elector and King, London, Thames \& Hudson, 1978. 
et la révolte échoue 3 . Pour l'heure, la paix intérieure du royaume est rétablie, mais, étant donné l'impopularité personnelle de George ler qui ne cache pas sa préférence pour son territoire allemand et ne parle que quelques mots d'anglais, et les forces toujours affectives, à défaut d'être militaires, du mouvement jacobite, personne ne peut supposer que la paix sera perpétuelle.

En France, la situation politique, quoique moins perturbée que celle de la Grande-Bretagne, ne va pas sans problème. Le jeune âge de Louis XV en 1715 rend une régence obligatoire ; et, si, finalement, le Parle-ment de Paris écarte certaines clauses du testament de Louis XIV pour reconnaître Philippe, duc d'Orléans, comme régent avec les pleins pouvoirs, cette décision est précédée d'intrigues et de négociations difficiles ${ }^{4}$. Autre observation : la santé faible de Louis XV amène à des inquiétudes politiques, car, s'il venait à mourir, la succession, comme en GrandeBretagne, serait disputée, cette fois entre le roi d'Espagne et le duc d'Orléans. Et qui sait si une lutte armée ne surviendrait pas ? Devant de telles incertitudes, le Régent adopte pour gouverner le système de la « polysynodie $»^{5}$.

Craignant l'éventualité de nouvelles instabilités poli-tiques, les gouvernements français et britannique rompent avec les traditions qui dominaient leurs relations diplomatiques depuislaseconde moitié dusiècle précédent, et signent en 1716 une alliance, à laquelle les ProvincesUnies et l'Autriche s'associent ${ }^{6}$. Dans un tel contexte, rendu encore plus difficile par l'affaire Law en France ${ }^{7}$ et par la crise de la South Sea Bubble en Angleterre ${ }^{8}$, on peut s'interroger sur la manière dont les gouvernements mettent les sciences au service économique de l'État.

Avant d'examiner la réponse apportée en GrandeBretagne à cette question, il faut souligner que, depuis la révolution de 1688 , le pouvoir du Parlement s'est beaucoup accru par rapport à celui du gouvernement. C'est le Parlement qui a annoncé «l'abdication » de

3 Bruce Lenman, The Jacobite risings in Britain, 1689-1746, London, Eyre Methuen, 1980 ; Murray Pittock, Jacobitism, Basingstoke, MacMillan, 1998; Daniel Szechi, The Jacobites, Britain and Europe, 1688-1788, Manchester, MUP, 1994.

4 On peut suivre ces négociations dans Jean-Christian Petitfils, Le Régent, Paris, Fayard, 1986, pp. 233-244, et Christine Pevitt, The man who would be king: the life of Philippe d'Orléans, Regent of France, London, Weidenfeld \& Nicolson, 1997, chap. 14.

5 Ce système est analysé dans Michel Antoine, Le cœur de l'État : Surintendance, contrôle général et intendances des finances, 1552-1791, Paris, Fayard, 2003, pp. 387-390.

6 Jeremy Black, British foreign policy in the age of Walpole, Edinburgh, Donald, 1985.

7 Antoin E. Murphy, John Law: economic theorist and policy maker, Oxford, Clarendon Press, 1997.

8 Richard Dale, The first crash: lessons from the South Sea Bubble, Princeton, Princeton University Press, 2004.
James II et qui a décidé les conditions de la succession de Marie et de Guillaume, comme il le fait à nouveau, en 1714, dans le cas de George ler. Le corps des parlementaires s'est trouvé renforcé à la suite de l'union avec l'Écosse, les quarante-cinq membres écossais faisant passer le nombre total des parlementaires de 513 à $558^{9}$. Et, depuis la fin du XVII e siècle, deux partis politiques - les Whigs et les Tories - se sont développés au Parlement, $d^{\prime}$ 'où ils influencent la politique de la Couronne ${ }^{10}$.

\section{En Grande-Bretagne, la prépondérance de l'initiative parlementaire}

Il faut constater que le gouvernement n'a guère de politique concernant les sciences dans leurs relations avec la vie économique. Son projet le plus important est annoncé en juillet 1714, donc avant l'avènement de George ler : il s'agit d'un prix de $20000 \mathrm{f}$ offert pour la découverte d'une méthode permettant de déterminer la longitude sur mer. Pour juger les projets, un comité est crée, le Board of Longitude, dont un des membres est le président de la Royal Society ${ }^{11}$.

En revanche, à la différence du gouvernement, des membres du Parlement sont à l'origine d'un corpus important de législation concernant l'économie ${ }^{12}$. Ainsi, ce sont les parlementaires, plutôt que la Couronne, qui sont les initiateurs des mesures tendant à appliquer les connaissances scientifiques à la vie économique ${ }^{13}$. Cette observation peut s'appuyer sur quelques chiffres. Entre 1714 et 1754, le Parlement vote 1493 lois et décrets, dont 723 - presque la moitié - sont classés " privés », ce qui signifie qu'ils ont été proposés par des membres du Parlement, et non par le gouvernement ${ }^{14}$. La plupart de ces lois et décrets, peut-être $75 \%$, sont à caractère économique ; ils cherchent soit à protéger la production, soit à accorder des privilèges à des producteurs qui ont convaincu les membres du Parlement que leurs inventions ou autres idées pour améliorer la production

9 Richard Romney Sedgwick, The history of Parliament: the House of Commons 1715-1754 I: Introductory survey, ap-pendices, constituencies, members A-D, London, HMSO, 1970, p. 3.

10 John P. Kenyon, Revolution principles: the politics of party, 1689-1720, Cambridge, CUP, 1977.

11 Sir Henry Lyons, The Royal Society, 1660-1940, Cambridge, Cambridge University Press, 1944, p. 144-145; sur la question de la longitude, Dava Sobel, Longitude: the true story of a lone genius who solved the greatest scientific problem of his time, London, Fourth Estate, 1996.

12 Phyllis Deane, The first industrial revolution, Cambridge, CUP, 1965, chap. 13.

13 Sir W. Holdsworth, A history of English law, London, Methuen, 1902-1972, 17 vol., vol. 11, p. 625-626.

14 R. R. Sedgwick, The History of Parliament, op. cit. note 9, p. 5. 
étaient dignes de leur soutien. Parmi les lois et décrets touchant l'économie, on a estimé que $42 \%$ traitaient du commerce extérieur, 20\% de l'agriculture, 19\% du commerce intérieur, $16 \%$ des manufactures et $3 \%$ des mines et carrières ${ }^{15}$. Évidemment, le commerce, avec un total de $61 \%$, occupe le premier rang, mais plus de deux cents lois enregistrées par le Parlement entre 1714 et 1754 abordent des questions ayant trait à l'agriculture, aux manufactures et aux mines et carrières.

Une conséquence importante de cette particularité de la législation est qu'elle ne peut constituer une stratégie économique nationale d'envergure. Les parlementaires qui présentent ces lois agissent à titre individuel, pour leurs propres régions ou localités. Leurs horizons politico-géographiques sont très limités. Au gouvernement, aucun ministre n'a la responsabilité de développer un programme national destiné, entre autres, à appliquer les sciences à l'économie. Le développement que connaissent l'agriculture, les manufactures ou I'exploitation des ressources minières en Grande-Bretagne pendant les premières décennies du xviiie siècle, est le fruit des efforts de particuliers au Parlement, et non de ceux du gouvernement.

\section{En France, l'implication profonde de l'État}

En France, la situation est très différente, pour trois raisons au moins. D'abord, le royaume a hérité du règne de Louis XIV un pouvoir étatique central fort ; les parlements, du moins à cette époque, ne sont pas en situation de faire échec à la politique royale, et la noblesse a été mise en coupe réglée. Ce pouvoir central prend des décisions qu'il veille à faire exécuter en s'appuyant sur une organisation administrative pyramidale, dont les principaux agents, les intendants, agissent comme de véritables chefs de I'administration régionale ${ }^{16}$. Ils ont pour mission de mettre en œuvre les ordres du roi sur le terrain. Constituant un maillage serré du territoire, ils relaient les décisions du pouvoir central jusque dans les campagnes les plus reculées de leur généralité, notamment par le biais des subdélégués et des notables locaux, et inversement font remonter à Versailles les informations les plus locales. Enfin, l'État dispose d'une institution scientifique officielle : I'Académie royale des sciences, qui a reçu une organisation fixe par le Règlement de 1699 et a été confirmée comme assemblée savante au service du pouvoir royal ${ }^{17}$.

15 Julian Hoppitt, A land of liberty? England 1689-1727, Oxford, Oxford University Press, 2000, p. 341, tab. 12.

16 Sur les intendants, leur rôle et leurs pouvoirs, Pierre Clément Timbal et André Castaldo, Histoire des institutions publiques et des faits sociaux, Paris, Dalloz, 9e éd. 1993, pp. 322-324.

17 Sur le règlement de 1699 et ses conséquences pour l'Aca-
Aussi, quand le nouveau Régent Philippe d'Orléans, investi le 2 septembre 1715, s'inquiète du mauvais état de l'économie française, il dispose de tous les instruments pour agir : sur le plan administratif, il peut s'appuyer sur le réseau des intendants auxquels il va s'adresser directement par des mémoires auxquels ils ont I'obligation de donner suite. Lui-même est fermement convaincu (conviction qu'il a héritée de son maître en chimie Guillaume Homberg) que les sciences doivent être mises au service de l'économie et des arts, donc de l'État. Et il a à sa disposition l'instance d'expertise savante à laquelle il va pouvoir confier la direction scientifique des opérations.

Ainsi, dès la fin de cette même année 1715, il lance une grande enquête nationale destinée à réunir des informations à la fois quantitatives et qualitatives sur les richesses naturelles de la France, et qui va s'intéresser particulièrement aux ressources minérales qu'on pourra mettre en valeur pour aider à relever l'économie nationale. Cette investigation va être menée tambour battant, en trois années, dans l'ensemble du royaume; aucune subdivision administrative, jusqu'aux plus éloignées, n'est laissée de côté.

Dans les faits, l'engagement de l'Académie des sciences dans ce qui sera connu ensuite comme l' « Enquête du Régent » est effectif, même si on en parle peu au cours de ses séances et si les procès-verbaux de la Compagnie n'en portent guère la trace. Néanmoins, quand on lit les très nombreux documents qui en ont été conservés dans les archives de l'Académie des sciences ${ }^{18}$, on ne peut douter de la réalité de son implication.

Outre le Régent lui-même, l'enquête est le fait de deux hommes: I'abbé Jean-Paul Bignon, qui est conseiller personnel auprès du Régent et membre influent de l'Académie des sciences ${ }^{19}$, et surtout un membre géomètre de l'Académie, René-Antoine Ferchault de Réaumur ; celui-ci, qui a déjà manifesté un grand intérêt pour les questions « techniques », va être

démie des sciences, Christiane Demeulenaere-Douyère et Éric Brian éd., Règlement, usages et science dans la France de l'absolutisme, Paris, Tec\&Doc, 2002.

18 Les documents issus de l'enquête y constituent aujourd'hui une masse considérable de documentation, intéressante à plus d'un titre, dont une édition critique vient d'être publiée : Christiane Demeulenaere-Douyère et David J. Sturdy, L'enquête du Régent, 1716-1718. Sciences, techniques et politique dans la France pré-industrielle, Turnhout, Brépols, 2008.

19 Neveu de Pontchartrain, «protecteur » officiel de l'Académie des sciences, il est le représentant de celui-ci auprès de l'Académie et le rédacteur du Règlement de 1699 ; ses liens avec les autres académies sont aussi fort étroits ; Chr. Demeulenaere-Douyère et D. J. Sturdy, L'enquête du Régent, op. cit. note 18, pp. 23-25. 


\section{Les sciences au service de l'économie en Grande-Bretagne et en France au début du XVIIIe siècle}

la véritable cheville ouvrière de l'expertise scientifique ${ }^{20}$, même s'il se fait parfois aider par d'autres académiciens (Lémery ou Geoffroy).

Réaumur trouve d'ailleurs dans cette enquête un intérêt personnel direct. Au même moment, il est engagé dans un autre projet académique, la Description des arts et métiers. Ce projet déjà ancien vise à publier un ouvrage à caractère encyclopédique qui doit réunir des savoirs et des savoir-faire sur les arts, avec le but de les diffuser auprès des artisans et des manufacturiers. Ainsi, Réaumur voit d'autant plus d'avantages à l'enquête de 1716-1718 qu'elle est pour lui un moyen de réunir rapidement et aisément des informations dont il a besoin pour cet ouvrage.

Enfin, au-delà de son expertise savante, l'Académie des sciences va apporter un appui logistique capital à l'enquête en lui ouvrant les portes de son laboratoire, où les échantillons de minerai envoyés par les intendants seront analysés et titrés.

L'enquête débute avec la diffusion d'un long mémoire, «Projet de lettre circulaire de Mgr le duc d'Orléans à MM. les Intendans, pour appuyer les mémoires des recherches que SAR a chargé I'Académie des sciences de suivre dans les différentes provinces du royaume par rapport à la physique et aux arts ${ }^{21} »$, rédigé par l'abbé Bignon avec vraisemblablement l'avis de Réaumur (on y retrouve certaines formulations usuelles sous sa plume). Des instructions précises et très détaillées y sont données aux intendants sur les objectifs de l'enquête et sur le mode opératoire de celle-ci. Le mémoire développe les principaux objets sur lesquels les intendants auront à recueillir des renseignements; on leur demande également de collecter des échantillons pour les envoyer à l'Académie des sciences qui les examinera et les soumettra à diverses expériences et analyses.

La circulaire est adressée progressivement à l'ensemble des intendants du royaume. Ce premier mémoire général est ensuite rappelé régulièrement à leur attention par des instructions particulières, rédigées par Réaumur.

Réaumur a préparé très scrupuleusement l'enquête, en faisant établir des extraits des mémoires qui avaient été collectés à la demande du duc de Beauvilliers, « pour l'instruction du duc de Bourgogne », en 1698 ; il étudie et annote soigneusement ces extraits et s'appuie sur ces dépouillements pour formuler ses premières demandes; il travaille aussi à partir de quelques ouvrages de référence pour rassembler des renseignements ${ }^{22}$.

20 Ibid., pp. 25-27.

21 Arch. Académie des sciences, Paris, pochette de séances, 1716 ; publié dans Chr. Demeulenaere-Douyère et D. J. Sturdy, L'enquête du Régent, op. cit. note 18, "Les origines de l'enquête », doc. 1.

22 Par exemple, Jean-Papire Masson (ibid., "Les origines de
À réception de la circulaire, les intendants n'ont plus qu'à se mettre au travail et à commencer à préparer leurs réponses, d'autant que les demandes sont faites au nom du Régent et qu'ils n'ont guère d'autre choix que d'y répondre le plus fidèlement qu'ils peuvent. Mais leur enthousiasme n'est pas toujours sans faille; certains répondent rapidement, d'autres se montrent moins zélés.

Les intendants adressent à Paris leurs premières données, en les accompagnant parfois d'échantillons de matières minérales, de dessins et de plans, et même ce qui atteste chez certains d'un esprit "scientifique » - de modèles en bois, terre cuite ou carton de certaines installations comme les fours ${ }^{23}$. Le Régent, qui prend connaissance personnellement de certains mémoires, transmet tous ces matériaux à Bignon ou, le plus souvent, à Réaumur. II se constitue ainsi un véritable circuit de l'information qui circule depuis les «informateurs» les plus obscurs sur le terrain jusqu'au Régent et à l'Académie des sciences, à Paris, en passant par les intendants qui en coordonnent la collecte au plan local et la transmission vers le pouvoir central.

L'intervention de Réaumur dans l'enquête, et par là l'expertise de l'Académie des sciences, est capitale. Réaumur exécute en effet un travail très méticuleux de compilation et de critique des données reçues; avec un soin sans égal et une patience infinie, il examine tous les documents qui lui parviennent, pièce à pièce ; il les analyse, les compare, les critique, les confronte avec les renseignements déjà recueillis par ailleurs, notamment dans ses lectures ou dans d'autres mémoires. II compare les données apportées par les mémoires des intendants ou des informateurs locaux et celles que fournissent les dessins ${ }^{24}$ et débusque les contradictions ou les incohérences.

Un premier mémoire donne rarement entière satisfaction par son exhaustivité et Réaumur a toujours de nouvelles questions à poser; d'ailleurs, un nouveau

I'enquête », doc. 3) et Pierre Borel (ibid., "Languedoc », doc. 12). 23 Ainsi, l'intendant de Navarre et Béarn, Legendre de Lormoy : « Je prends aussi la liberté de joindre à ma lettre [...] un dessein du four où l'on a fait cuire les pierres. J'en ay aussy fait faire un modèle en carton que j'ay mis dans le balot pour donner à l'académie une connoissance plus exacte de l'intérieur du fourneau. » (ibid., "Navarre et Béarn », doc. 20) ; voir aussi ibid., "Lyon », doc. 16. II ne subsiste malheureusement aujourd'hui aucun de ces modèles.

24 Sur le rôle du dessin, Christiane Demeulenaere-Douyère, «Enquête administrative et dessin technique dans la France préindustrielle : I'exemple de l'enquête du Régent, 1716-1718», dans Christiane Demeulenaere-Douyère, Martine Plouvier et Cécile Souchon dir., Des images et des mots. Les documents figurés dans les archives, Actes du $132^{e}$ congrès national des sociétés historiques et scientifiques, Arles, 2007, Paris, Éd. du CTHS, à paraître. 


\section{Christiane Demeulenaere-Douyère \& David J. Sturdy}

mémoire suscite souvent de sa part de nouvelles curiosités. Il envoie aux intendants ses demandes d'éclaircissements, toujours au nom du Régent, réclame des explications, des dessins exacts et non pas « bien finis », munis d'une échelle. Les intendants doivent alors se mettre à nouveau en quête d'informations complémentaires et rédiger de nouveaux mémoires, présentés souvent en colonnes, les réponses de l'intendant faisant face aux questions de I'Académie et leur répondant point par point ${ }^{25}$.

Si Réaumur n'est pas encore pleinement satisfait des éclaircissements nouveaux donnés par les intendants, il revient à la charge, toujours avec une exquise courtoisie ${ }^{26}$, mais avec fermeté, au risque de lasser parfois la bonne volonté des intendants. Pour eux, il n'est pas forcément aisé de rassembler les informations demandées, d'autant qu'ils sont chargés d'autres tâches administratives, très nombreuses et fort accaparantes. Eux-mêmes sont tributaires de leurs propres "informateurs», plus ou moins zélés à les aider rapidement. ॥ faut trouver les interlocuteurs compétents et fiables; certains sont imprécis ${ }^{27}$, d'autres très imaginatifs et donnent des renseignements plus ou moins fantaisistes qu'il faut ensuite aller contrôler sur le terrain ${ }^{28}$. Des obstacles matériels peuvent aussi ralentir les progrès de l'enquête ; ainsi, dans les régions montagneuses, les mines dont on veut tirer des échantillons sont souvent difficiles d'accès, situées dans des endroits escarpés, inaccessibles en hiver à cause de la neige ${ }^{29}$. Par ailleurs, alors que beaucoup $d^{\prime}$ '« informateurs » acceptent d'apporter leur concours à l'enquête sans réticences, certains refusent de collaborer

25 Par exemple, Chr. Demeulenaere-Douyère et D. J. Sturdy, L'enquête du Régent, op. cit. note 18, "Navarre et Béarn », doc. 20.

26 Réaumur écrit par exemple de l'intendant de Lyon: "Nous ne nous lassons point de demander des instructions de la généralité de Lion. Les excellents mémoires que Monsieur Méliand nous a procuré nous font même souhaiter d'avoir souvent des occasions de luy demander » (ibid., "Lyon », doc. 10).

27 Pourtant fort zélé à servir l'enquête, l'intendant de Lyon écrit : " Je demande mil excuses à VAR sy j'ay tant tardé à luy envoyer les éclaircissements qu'elle m'a fait l'honneur de me demander sur plusieurs articles du premier mémoire que j'ay pris la liberté de luy adresser pour l'académie des Sciences, mais c'est un ouvrage qui n'a pas dépendu de moy seul. II a falu tirer des connoissances de différentes personnes et s'assujettir à leur peu d'exactitude " (ibid., " Lyon », doc. 7).

28 Ainsi, en Auvergne: "Ces mémoires ont été dressés par un homme de la province, lequel a beaucoup d'esprit et de littérature, mais, comme il ne s'est pas donné la peine de se transporter sur les lieux pour voir et examiner par luy même les choses rares et curieuses qui sont répendues dans son mémoire, on ne peut ajouter une foy entière aux faits qui y sont avancés » (ibid., "Riom ou Auvergne », doc. 5).

29 Ibid., « Perpignan », doc. 4. malgré des injonctions officielles ${ }^{30}$. Mais, malgré tous ces obstacles, l'enquête du Régent est achevée en trois années, au prix, il est vrai, d'un travail le plus souvent très considérable.

Avec les mémoires, sont aussi adressés à Paris de nombreux échantillons de matières minérales (or, argent, fer, étain, plomb), des pierres précieuses ou non (turquoises, améthystes, marbres, craies), des coraux et autres produits de la mer (dont le transport pose des problèmes matériels délicats), des sables, des perles, des eaux minérales, voire du pétrole...

Essayer tous ces échantillons qui arrivent presque chaque semaine en paquets, boîtes, sacs, etc., est du ressort de l'Académie, et plus exactement de son laboratoire où œuvre Réaumur, qui s'adjoint les services d'un certain Fousjean, dont on sait seulement qu'il s'est occupé d'exploitation minière en Alsace. Ce gros travail ne va pas sans difficultés. II arrive que la quantité de matière fournie soit insuffisante pour permettre des essais et Réaumur ou Fousjean sont obligés de réclamer. D'autres fois, les échantillons qui arrivent à l'Académie portent des étiquettes erronées : un intendant a désigné une matière comme "argent», alors que les essais faits à Paris montrent qu'il s'agit d'autre chose ; il faut donc prendre le temps de vérifier la véritable nature des matières reçues. Réaumur et Fousjean traitent les échantillons aussi vite qu'ils peuvent, mais, en janvier 1719 (donc après la fin de l'enquête), ils n'ont pu achever leurs essais que sur des échantillons de minerais provenant de moins de la moitié des trente et une généralités concernées par l'enquête ${ }^{31}$.

Une question s'impose : au terme de tout ce processus, à quel résultat est-on parvenu ? L'enquête a permis de rassembler une documentation abondante et riche d'informations précises sur l'économie du royaume de France et sur ses possibilités de développement. Sous l'influence de Réaumur, les investigations ont été plus poussées dans le domaine de l'exploitation minière ${ }^{32}$ et dans celui de l'industrie, particulièrement

30 Ibid., "Caen », doc. 5-10.

$31 \mathrm{La}$ liste en est la suivante: Alsace (9 mines), Bourgogne (4), Languedoc, Toulouse et Montpellier (8), Dauphiné (26), Auvergne (2), Limoges (3), Duché de Bourgogne (1), Poitiers (2), Perpignan pour le Roussillon (1), Lyon (9), Châlons pour la Champagne (1), Bretagne (6), Béarn et Basse-Navarre (25), Aix pour le Provence (7) (D. J. Sturdy, "L'Académie des sciences et I'enquête du Régent de 1716-1718», dans Chr. DemeulenaereDouyère et É. Brian éd., Règlement, usages et science, op. cit., pp. 139-140).

32 Christiane Demeulenaere-Douyère, « Une tentative d'inventaire des richesses minières de la France : I'enquête du Régent, 1716-1718», Documents pour I'histoire des techniques, 16, 2008, pp. 9-16. 


\section{Les sciences au service de l'économie en Grande-Bretagne et en France au début du XVIIIe siècle}

métallurgique, et il y aura une étude intéressante à entreprendre sur l'évaluation des résultats de l'enquête.

Mais, en raison de circonstances particulières (majorité de Louis XV, puis mort du Régent en 1723), ce gigantesque chantier n'a pas été suivi d'effets politiques immédiats et visibles. L'enquête semble même être tombée dans un relatif oubli administratif. Sur le plan scientifique aussi, si Réaumur a utilisé certaines informations obtenues dans le cadre de l'enquête pour ses travaux personnels ou pour la documentation de son Art de convertir le fer forgé en acier... (publié en 1722), il semble s'en être assez vite détourné pour d'autres centres d'intérêt scientifique.

Toutefois, née de la seule volonté du pouvoir royal, orchestrée par lui dans ses moindres détails et relayée et exécutée par ses agents, I'enquête du Régent est importante à plus d'un titre. Elle marque une étape nouvelle, en France, dans la mise au service de l'utilité publique des sciences et des techniques, constituant ainsi un précédent aux grandes enquêtes scientifiques de la fin du XVIIIle siècle (sur les hôpitaux de Paris, sur les abattoirs...) dans lesquelles l'Académie royale des sciences bénéficiera pleinement du statut de partenaire scientifique de l'État. De plus, en contribuant à inscrire des critères d'ordre "scientifique » ou «technique» au rang des considérations que les administrateurs du royaume doivent prendre en compte dans la gestion, notamment économique, des territoires confiés à leurs soins, elle participe à l'élaboration de méthodes modernes d'administration.

Ce regard rapide porté comparativement sur la Grande-
Bretagne et la France, à la sortie d'une longue période de guerres, suggère au moins quatre conclusions.

En premier lieu, dans les deux pays, les gouvernements (avec, en Grande-Bretagne, les membres du Parlement) acceptent la nécessité de se consacrer à la recherche de la prospérité économique, ce qui contraste de manière frappante avec les décennies précédentes, où les gouvernements sacrifiaient la prospérité nationale aux exigences de la guerre.

Par ailleurs, on voit qu'en Grande-Bretagne, la plupart des initiatives procèdent des parlementaires plutôt que du gouvernement ; en conséquence, il est difficile, voire impossible, de discerner la naissance d'une stratégie économique nationale. En France, c'est le contraire: l'enquête du Régent témoigne d'une volonté réelle de la part du gouvernement, à la fois, d'imaginer une telle stratégie et de tenter une expérience d'envergure qui peut contribuer à sa réalisation.

Les chiffres des Acts du Parlement qu'on a cités plus haut indiquent qu'en Grande-Bretagne, on privilégie le commerce et l'agriculture comme moteurs principaux de la restauration économique, tandis qu'en France, l'enquête du Régent met en évidence un intérêt plus marqué pour l'exploitation des ressources minérales et la mise en valeur des mines.

Alors qu'en Grande-Bretagne, les sciences en général et la Royal Society en particulier ne contribuent à la restauration économique qu'aux marges, en France, à la même époque, les sciences et l'Académie royale des sciences se trouvent placées dans un rôle central. 